\title{
PERFORMANCE OF APN GUIDANCE LAW IN A TWO DIMENSIONAL AERIAL ENGAGEMENT SCENARIO
}

\author{
K.N.Swamy, Scientist, Advanced Technology Programme and \\ I.G.Sarma, Professor of Computer Science and Automation \\ Indian Institute of Science, Bangalore, India.
}

\section{Abstract}

In the available literature, the main advantage claimed for APN is that it demands a progressively decreasing missile acceleration in the constant target maneuver situation, as opposed to an increasing missile acceleration for the more conventional PN guidance. This inference is mainly based on studies with one dimensional models. The present study, based on a two dimensional model, not only incorporates a more realistic target maneuver, but also considers the effect of target glint characterised by correlated noise. The missile acceleration and the miss-distance induced by target maneuver and glint are the two features of performance investigated in this study. In the deterministic study, with target maneuver initiation occuring at various points in time, the acceleration profiles resemble the ones obtained from a linear quadratic tracking formulation of the guidance problem. The results indicate that there is a considerable improvement in the miss distance performance over PN, for maneuvers which are initiated when the relative seperation is small.

\section{Introduction}

Classical guidance laws like the Proportional Navigation (PN) have been employed quite successfully in the past for homing guidance. In particular, using low pass filtering to attenuate the noise inherent in the guidance signal and applying the PN law to steer the missile towards the target works well in benign environments. However, in recent years the performance of threat aircraft have improved substantially in terms of speed and agility. The performance improvement required of PN to meet these additional demands increases the acceleration requirement of the air frame.

While there is a great deal of published literature on PN, that available on Augmented PN (APN) is scanty. The exhaustive survey paper by Pastrick et al [1] while discussing PN at length, hardly provides any information about APN.

The main advantage claimed for APN by Nesline and Zarchan [2] is that it demands a progressively decreasing missile acceleration in the constant target maneuver situation, as opposed to an increasing missile acceleration for the more conventional PN guidance. This is achieved by explicitly incorporating the target aceleration term in the APN guidance law. In other words, the commanded acceleration in APN has the same form as that in $\mathrm{PN}$, but for the extra additive term $1 / 2 \mathrm{~N}^{\prime} \mathrm{a}$ where, $N$ ' is the effective navigation ratio and $\mathrm{a}_{\mathrm{T}}$ is the target acceleration. The target and the missile are both assumed to have acelerations normal to the chosen reference direction. In the actual implementation of APN, there is the additional complexity of having to estimate the target maneuver on board the missile. The target models that are generally used in literature are those obtained by passing white noise through a shaping filter $[3,4]$. A step target maneuver with the time of initiation uniformly distributed over the engagement time is shown to correspond to white noise through an integrator [5].

The present study assumes a deterministic target maneuver model, represented by initial level flight, followed by the execution of a constant acceleration maneuver in the vertical plane. From among the various sources of noise which affect the performance, the effect of target glint characterised by correlated noise is investigated, since it is the most significant. The missile acceleration and the miss-distance induced by target maneuver and glint are the two features of performance investigated in this study.

The studies have been carried-out under the following conditions: one in which the target maneuver level and its initiation time are assumed to be known precisely and the other, wherein the target maneuver is estimated by a weighted least squares method with the attendant detection delay and estimation error. In addition to PN, a version of Linear Quadratic (LQ) guidance called MAMS [6] is also used for comparing the performance. The comparative acceleration profiles are discussed for PN, APN and LQ guidance schemes, and the miss distances for the PN and APN schemes.

\section{Models for target maneuver and glint}

The comparative performance study for the three guidance schemes requires development and use of suitable models for target maneuver and glint. The target maneuver model proposed here is deterministic involving a constant step acceleration in the vertical plane till it levels off for retreat. On the other hand, being a noise term, glint is modelled stochastically.

Target maneuver models

The problem of how to model target accelera- 
tion to achieve improved missile performance, has been extensively discussed in literature. This is a critical problem area for APN and LQ guidance implementations, since the estimation of missile-to-target position, velocity and acceleration depend on this.

In the target model proposed and used in this study, it is assumed that the target flies level till time $t_{\text {man }}$ and then executes a constant maneuver $a_{T}$ till it levels-off for a retreat as shown in Fig. 1. This model is justified from practical considerations, since the target generally pulls a maximum maneuver and retreats after delivery of its weapon load. The maneuver is detected and estimated on board the missile after its occurrence at $t$. The magnitude of target velocity $v_{T}$ is assumed to be a constant.

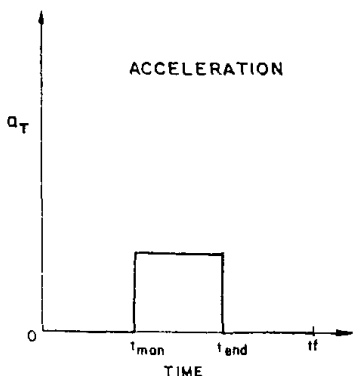

(0)

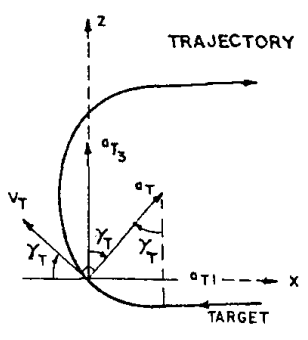

(b)
FIG. 1 LEVEL AND STEP TARGET MANEUVER

\section{Glint Noise}

Glint is correlated noise which is represented by passing white noise through a shaping filter. The equivalent white noise spectral density is given by [3]

$$
\phi_{g}=2 \tau_{g} \sigma_{g}^{2}
$$

Where $\tau_{g}$ and $\sigma_{g}$ are the correlation time constant and standard deviation of glint noise respectively. Representative values for these parameters [3] are $\tau_{\mathrm{g}}=0.1 \mathrm{sec}$. and $\sigma_{\mathrm{g}}=3 \mathrm{met}$.

Following [7], to generate white noise of spectral density ( $2 \tau_{g} \sigma$ ), a white sequence of variance $\sigma^{2}$ is generated with a spacing of $\boldsymbol{\Delta}$. Assuming that the correlation of the white sequence drops to zero over $\Delta$, a triangular correlation is obtained. Since for small $\Delta$, spectral density is approximately the area under the correlation curve, we have for the white sequence generated

\section{Spectral density $\phi=\Delta \sigma^{2}$}

Equating this to the equivalent glint white noise spectral density, we see that the white sequence generated will have the variance given by

$$
\sigma^{2}=2 \tau_{g} \sigma^{2} / \Delta
$$

\section{PN Guidance}

Traditionally, homing intercept problems have been solved through the use of PN. This form of guidance law commands a missile acceleration which is proportional to the line-of-sight (LOS) rate and the proportionality factor is called the Navigation ratio. The engagement geometry is shown in Fig.2. The commanded acceleration is given by

$$
a_{c}=N^{\prime} v_{c} \dot{\lambda}
$$

where

$v_{c}=$ Closing velocity and

$N^{\prime}=$ Effective navigation ratio

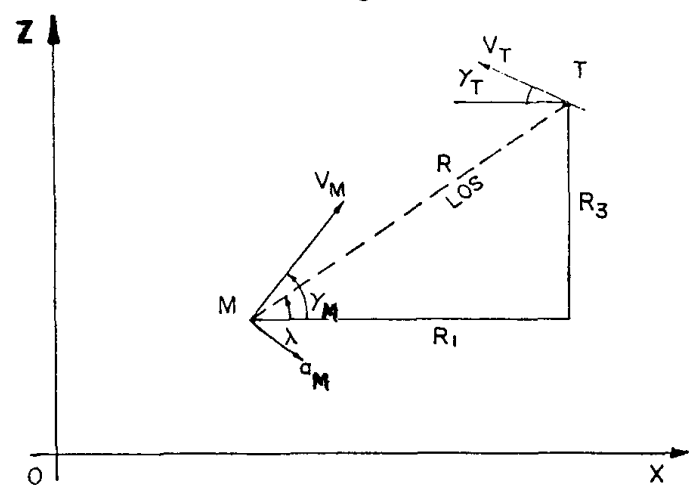

\section{FIG. 2 ENGAGEMENT GEOMETRY}

The principal outputs of the missile seeker are the dish rate which is a measure of the line-of-sight rate, and the Doppler frequency which is proportional to the closing velocity. The dish rate which is contaminated by glint noise is passed through a noise smoothing filter [2] to obtain the commanded acceleration as shown in Fig.3.

\section{Choice of effective navigation ratio}

The performance of the missile is first considered against maneuvering targets for different maneuver initiation times, for values of navigation ratio $N^{\prime}$ in the range 3 to 5 . Results for the deterministic case indicate that the miss distances decrease with increasing $\mathrm{N}$ '. The missile accelerations are larger initially for $\mathrm{N}^{\prime}=5$ compared toN' $=3$ or 4 . A comparison of results for various $N^{\prime}$ in the range 4 to 8 for the stochastic case (with glint noise and filter) reveals that the commanded acceleration becomes very large and noisy for $N^{\prime}=8$, essentially due to the amplification of glint noise. Based on the above studies, a compromise value of 5 is chosen for $\mathrm{N}$. 


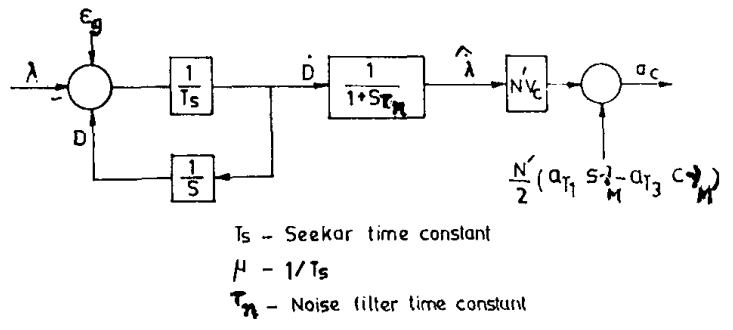

FIG. 3 SIMPLIFIED SCHEMATIC OF IHE SEEKER LOOP

\section{Noise filter time constant}

Since glint noise has been modeled as a white noise process through a first order filter of frequency $10 \mathrm{rad} / \mathrm{sec}$, it essentially contains low frequency components. A low-pass filter of frequency $5 \mathrm{rad} / \mathrm{sec}\left(\tau_{n}=0.2 \mathrm{sec}\right)$ has been introduced to filter out the dish rate noise. This value has been chosen after studying the performance of filters with different time constants over a range of 0.1 to $0.5 \mathrm{sec}$. The mean miss distances at various maneuver initiation times have been investigated. The smallest miss distance has been obtained for a filter time constant of $0.2 \mathrm{sec}$. The filter with $\tau_{n}=0.2 \mathrm{sec}$, permits the signal to pass through without much attenuation and cuts of the noise effectively. From these considerations, the time constant of $0.2 \mathrm{sec}$ is chosen.

\section{APN Guidance}

\section{APN Law}

The PN guidance law

$$
a_{c}=N^{\prime} v_{c} \dot{\lambda}
$$

can also be expressed as [2]

$$
a_{c}=\left(N^{\prime} / t_{g o}{ }^{2}\right)\left(y+\dot{y} t_{g o}\right)
$$

where $y=$ missile target separation normal to reference direction

$\mathrm{R}=\mathrm{Vctgo}$

$\mathrm{V} C=$ closine speed (constant)

$$
\lambda \simeq Y / R,
$$

If the target accelaration $\mathrm{a}_{\mathrm{T}}$ is considered, the commanded accelaration becomes

$$
a_{c}=\left(N^{\prime} / t_{g o}{ }^{2}\right)\left(y+\dot{y} t_{g o}+1 / 2\left(a_{T} t_{g o}{ }^{2}\right)\right\}
$$

The guidance law given above is termed as Augmented PN. This differs from the PN in the inclusion of an extra term dependent on target accelation. Here the relative separation, relative velocity and target acceleration are considered only in one direction; namely normal to the reference direction. In the APN guidance equation developed here, the commanded missile acceleration of $\mathrm{PN}$ is augmented by an extra term which has $X$ and $Z$ components of target acceleration resolved normal to the missile.

\section{The APN Dynamic Model}

The target to missile relative separation components R1 and R3 are given by

$$
\begin{aligned}
& \dot{\mathrm{R}} 1=\mathrm{V}_{1} \\
& \dot{\mathrm{R}} 3=\mathrm{V}_{3}
\end{aligned}
$$

where $\mathrm{VI}$ and $\mathrm{V} 3$, the target to missile relative velocity components are given by

$$
\begin{aligned}
& \dot{v}_{1}=-a_{M} \sin \gamma_{M}+a_{T 1} \\
& \dot{v}_{3}=a_{M} \cos \gamma_{M}+a_{T 3}
\end{aligned}
$$

aTl and aT3 are the components of target acceleration, and $\mathrm{aM}$ is the missile acceleration.

From the engagement geometry of Fig.2. Hence,

$$
\tan \lambda=\mathrm{R}_{3} / \mathrm{r}_{1}
$$

$$
\dot{\lambda}=\left(R_{1} V_{3}-R_{3} v_{1}\right) /\left(R_{1}^{2}+R_{3}^{2}\right)
$$

Assuming that the dish rate $\dot{D}$ is proportional to the angular error including glint, we get

$$
\dot{\mathrm{D}}=-\mu\left(\varepsilon_{\mathrm{b}}+\varepsilon_{\mathrm{g}}\right),
$$

where $\boldsymbol{\varepsilon}_{\mathrm{b}}$ is the boresight error and $\boldsymbol{\epsilon}_{\mathrm{g}}$ is the angular glint noise and $\mu$ is a constant $(\mu=$ $1 / \zeta)$. Hence

$$
\dot{\mathrm{D}}=-\mu\left((D-\lambda)+\varepsilon_{\mathrm{g}}\right\}
$$

Where $\mathrm{D}$ and $\lambda$ are the dish angle and the LOS angle respectively. The flight path angle $\gamma_{M}$ is given by

$$
\dot{r}_{M}=-a_{M} / v_{M}
$$

and the missile acceleration by

$$
\dot{a}_{M}=\left(-a_{M}+a_{c}\right) / \tau_{a}
$$

assuming a first order autopilot lag of time constant $\tau_{a}$. The angular glint $\epsilon_{g}$ (Fig.4) is given by

$$
\dot{\varepsilon}_{L}=1 / \tau_{g}\left(-\varepsilon_{L}+w_{H}\right)
$$

where

$$
\varepsilon_{L}=R \varepsilon_{g}
$$

The output of the noise filter is given by

$$
|\hat{\dot{\lambda}}|^{\prime}=(\dot{D}-\hat{\dot{\lambda}}) / \tau_{n}
$$

which is an estimate of the LOS rate.

The expression for commanded acceleration is

$$
a_{c}=-N^{\prime}\left|v_{c}\right| \hat{\dot{\lambda}}+N^{\prime} / 2\left(a_{T_{1}} \sin \gamma_{M}-a_{T_{3}} \cos \gamma_{M}\right)
$$


The above set of equations provide the trajectory for the state variables $\mathrm{R} 1, \mathrm{R} 3, \mathrm{~V} 1, \mathrm{~V} 3$, $\lambda, \lambda_{M}, \mathrm{D}, \mathrm{a}_{M}, \epsilon_{\mathrm{g}}$ and $\widehat{\hat{\lambda}}$, where $\mathrm{a}_{\mathrm{T}}$ and $\mathrm{a}_{\mathrm{T}}$ are the components of target acceleration obtained from an assumed target trajectory and $\epsilon \mathrm{g}$ is the glint noise. The commanded acceleration ac is obtained as a function of state variables.

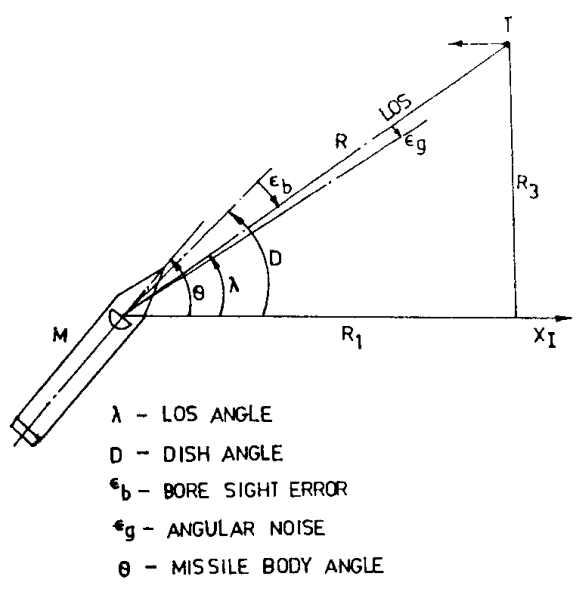

FIG. 4 GEOMETFY OF SEEKER

\section{LQ Guidance}

Starting from the late 1960's, several newer forms of guidance laws using optimal control and differential game theories have been proposed in literature [8]. Modern guidance and estimation theories would allow the design to "optimally" separate the signal from the noise by using information about the missile dynamics and noise covariances rather than filtering based only on frequency contents. The estimates are then used to generate the guidance signal (i.e., commanded acceleration) based upon a suitably specified optimality criterion. This performance index can be structured to include penalties on undesirable features like trajectory deviations, excessive accelerations etc. in addition to miss distance.

The most commonly advocated approach to tactical missile guidance is the one based on Linear-Quadratic-Gaussian theory. This formulation requires the development of a linear model for the engagement scenario and selection of a suitable performance index that results in an explicit form for the solution.

Incorporating model-tracking features and time-varying weights turns out to be critical for obtaining miss distance and acceleration performance superior to that of PN and APN.
In a companion paper by the authors [6] the reference relative trajectory is derived from a pulsed missile acceleration, which has been shown in literature to be time and energy optimal. The width and location of the pulse depends upon the engagement conditions, heading error and target maneuver. The height of the pulse could be varied according to the latax capability of the airframe. Heading error requires an initial pulse, while a constant $g$ target maneuver demands another reference pulse, coinciding with the maneuver initiation.

The vehicle acceleration follows the reference pulse closely in the optimal guidance scheme and the achieved acceleration remains small for a large portion of the flight time.

\section{Study Conditions}

Unlike PN, both APN and $L Q$ guidance need a target maneuver detection and estimation scheme on board the missle, since target maneuver terms appear explicitly in the guidance algorithm. A state model of planar engagement with a mesurement model employing the homing head and autopilot measurements is used for maneuver estimation. A Kalman filter algorithm is also employed for state estimation in MAMS. The presence of noise affects the commanded acceleration through the dish rate in PN, while it affects the measurement covariance in APN and LQ guidance scheme. The estimation of tgo is essential in LQ guidance scheme. PN and APN do not need this estimate.

\section{Maneuver detection and estimation}

A simplified Kalman filter denoted as SKF, which does not contain the maneuver term is basically used for maneuver detection and estimation. The method used follows the input estimation procedure of Chan et al [9]. In this scheme, the difference between the actual measurements and the state estimate from the SKF is obtained at succesive measurement points to estimate the target maneuver. Typically, ten samples of measurements are used to arrive at an estimate of target maneuver. A weighted least squares moving window algorithm is adopted. Succesive values of the estimate are used to make an assesment of the occurence of the maneuver by comparing them with a preset threshold value. A large departure of maneuver estimate from the threshold signals detection. To estimate the level of the maneuver itself, a variant of the method proposed in [9] is used. The proposed method uses incremental measurements by considering increments in the measurements over that at any chosen time point, after the detection has occured.

\section{Engagement Conditions}

Deterministic and stochastic cases are 
both considered. Only glint noise is included in the stochastic case. Perfect detection and estimation implying no detection and estimation errors, and noise-free dish rates are assumed in the deterministic runs. In the stochastic cases, detection and estimation procedures described earlier are used. Besides, the glint noise effect on the dish rate is also considered. The commanded acceleration is obtained by passing the dish rate through a noise filter in the stochastic cases. Both the cases of short and long range engagements are considered, with the target maneuvering at different points, to bring out their influence on the acceleration profile and miss distance. A maximum value of $6^{\circ}$ is arbitrarily specified for the heading error (HE). The missile acceleration capacity is assumed to be bounded. A fixed value of $8 \mathrm{~g}$ is used for target accleration.

\section{Comparative Performance}

The main features that are used for comparison in the deterministic and stochastic cases are (a) acceleration profiles and (b) miss distances in $\mathrm{PN}, \mathrm{APN}$ and $\mathrm{LQ}$ guidance implementations.

\section{Deterministic situation}

(a) The acceleration behaviour in the deterministic cases is shown in Figures 5 and 6 for early and late maneuver occurence situations, for zero heading error and long intercept range.It is observed from the figures that the acceleration is zero in all the cases till the occurence of maneuver, since the heading error is zero. When the maneuver occurs, the commanded acceleration in APN and MAMS show a sudden discontinuity unlike in $\mathrm{PN}$. This is because the target maneuver itself changes abruptly from 0 to $8 \mathrm{~g}$ and in the APN an extra term is added in ac to take account of the target maneuver, while in MAMS ac is obtained by using a pulsed acceleration for tracking, which is introduced suddenly because of target maneuver.

This way, in APN and MAMS a large amount of acceleration is added at the start of target maneuver itself. It is advantageous to turn the missile when the separation is large, since this would need small pitching motions. MAMS takes advantage of the predicted point of intercept and settles to a straight line course. APN also has a near similar behaviour even though there is no concept of a predicted intercept point in APN. The commanded acceleration in PN shows no such discontinuity since the target maneuver does not cause discontinuous dish rate changes. PN shows large values of ac with change of sign, since the acceleration follows the dish motion. The variation in acceleration after the completion of the pulse in APN is much smaller, while that in MAMS is the smallest. It is interesting to observe that the APN acceleration profile resembles the MAMS profile closely. The small acceleration requirement in APN makes the blind phase of the flight easy to cope, as in MAMS.

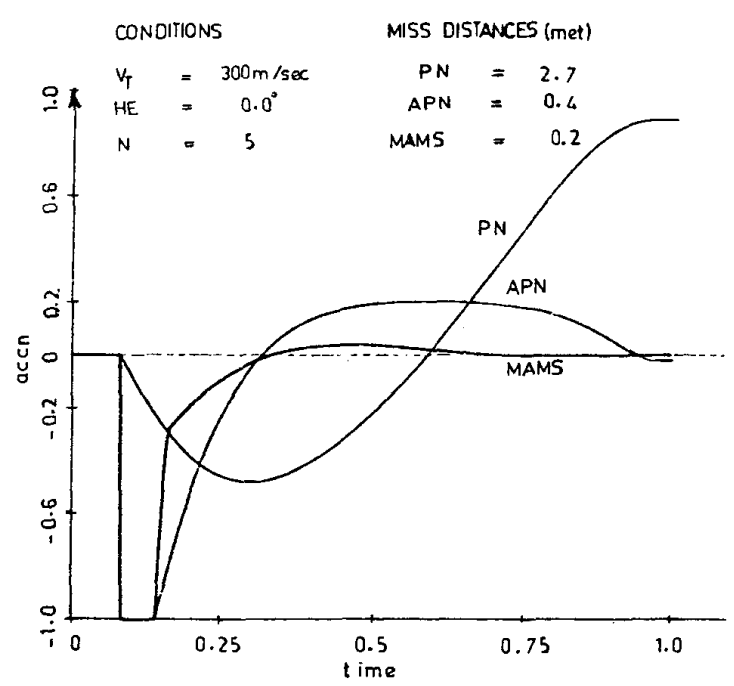

EIG. 5 Acceleration requirement comparison for early maneuver

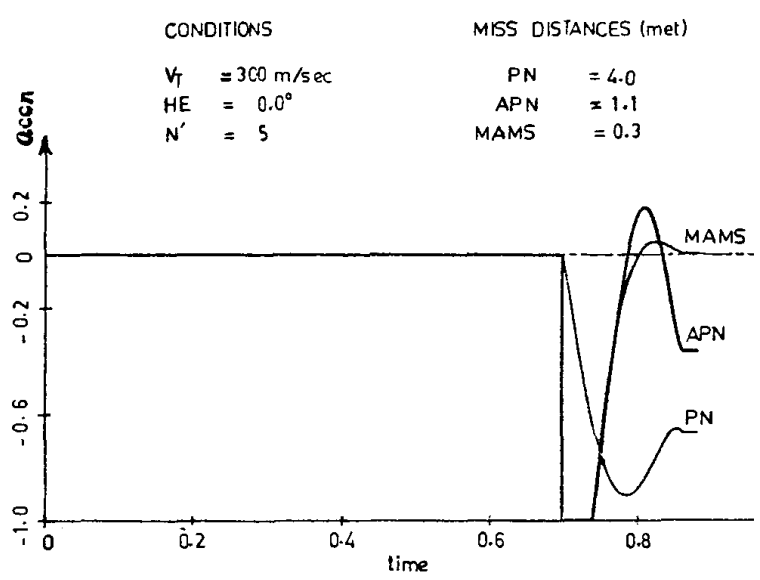

FIG. 6 Acceleration requirement comparison for late maneuver

In APN, the contribution initially of the target acceleration term to the commanded acceleration is of the same sign as that of dish rate (both being negative). Hence ac is a large negative quantity in APN, reaching saturation value. In $\mathrm{PN}$, the acceleration level undergoes large excursions. Further, the APN acceleration is more uniform around zero due to the combination of dish rate and the component of target acceleration, with the acceleration profile of MAMS representing the ideal situation. Thus the integrated square acceleration is smaller in APN compared to PN.

The presence of initial heading error modifies the initial portions of the acceleration profiles. 
In $\mathrm{PN}$ and APN it adds a positive acceleration initially, while in MAMS a positive initial pulsed acceleration is caused by $\mathrm{HE}$. HE however has no significant effect on the miss distance, particularly for late maneuver initiations.

(b) The miss distances for these engagement conditions are also shown in Figs 5 and 6 . A study of the miss distances for various $t$ reveals that in $\mathrm{PN}$, the miss distance is large for both small and large values of t $t$, while being very small in between these values. Also, for excessively delayed maneuvers and for level flight conditions, the miss distances are very small. APN also shows a similar performance but with maximum values of miss smaller than those in PN. The miss distances in MAMS could be reduced to small values in all the cases, by properly tuning the performance index weights and selection of reference trajectory.

\section{Stochastic situation}

While considering the stochastic cases, the results have been averaged over 50 montecarlo runs. The point of maneuver detection and the maneuver level vary from run to run. Mean and 16 values are shown in Tables 1 and 2 . It is seen from the individual runs that the detec:ion delay varies between 240 millisec and 500 millisec, the delay being larger for small maneuver levels. The accuracy of maneuver estimation is observed to be better than $6 \%$ in all these runs, for the assumed measurement noise covariance.

(a) The acceleration profiles in PN, APN and MAMS have the same pattern as in the deterministic cases, but now show excursions around the deterministic values due to the glint noise affecting commanded acceleration. The magnitude of these fluctuations around the deterministic profile increases terminally, due to the glint noise becoming more predominant towards the end of the engagement. (b) The mean and $1 \sigma$ miss distance values obtained for PN, APN, and MAMS are recorded in Tables 1 and 2 for short and long range cases respectively, with a heading error of $6^{\circ}$. Even when perfect information is assumed about the target maneuver, the situation becomes stochastic due to the noisy dish rates. From both the cases, it may be observed that the maximum values of miss which occur in PN for late maneuver cases are significantly reduced in APN. For early maneuvers, the APN miss distances tend to be larger than in $\mathrm{PN}$ in the short range case. In the long range case, very early initiation of maneuver causes a larger miss than in PN. This is because, the absence of the target acceleration term in APN causes an abrupt change in a when the target levels off for retreat. Comparison of the miss distances in PN and APN for the deterministic and stochastic cases reveals that they increase significantly - nearly by a factor of 4 - in the stochastic cases. Comparison is made with MAMS only for the critical PN cases. The miss distance values shown for MAMS are only for the assumed measurement covariances. For the other cases, the miss distances in MAMS are smaller than the PN and APN values.

\section{Conclusions}

As far as the miss distance is concerned, the maximum value obtained in APN is smaller than in PN for the range of maneuver initiation times investigated. The miss distances in the LQ guidance scheme can be made even smaller by proper selection of performance index weights and the reference nominal.

In the deterministic study, with the target maneuver initiation occuring at various points in time, the PN acceleration profiles exhibit large magnitude excursions of both polarities whereas in APN, the acceleration profile resembles a pulse, with the pulse height being the maximum permissible acceleration. Furthermore,

TABLE 1. MISS DISTANCE COMPARISON (SHORT RANGE CASE)

$$
\mathrm{HE}=6^{\circ}
$$

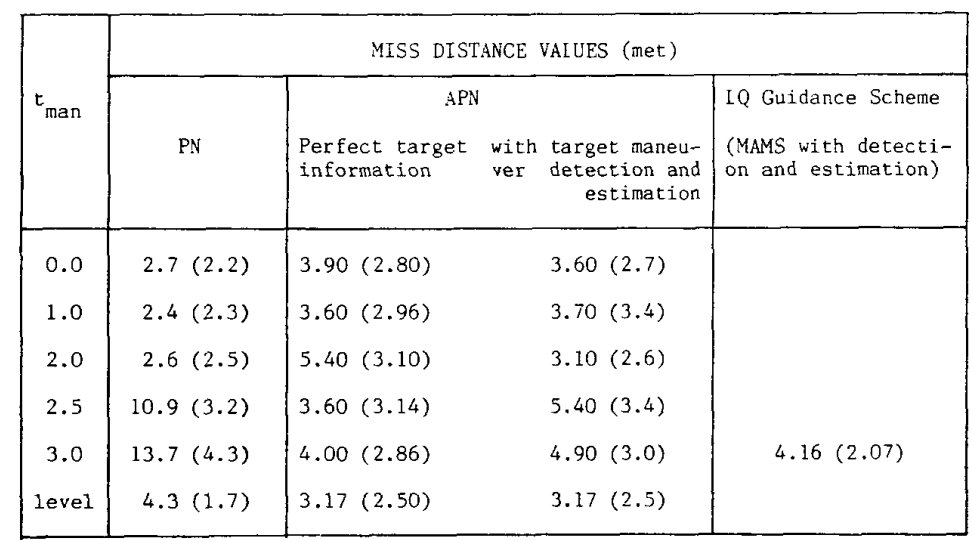


TABIE 2. MISS DISTANCE COMPARISON (IONG RANGE CASE)

$$
\begin{aligned}
H E & =6^{\circ} \\
N^{\prime} & =5
\end{aligned}
$$

\begin{tabular}{|c|c|lc|l|}
\hline \multirow{2}{*}{$t_{\text {man }}$} & \multicolumn{5}{|c|}{ MISS DISTANCE VALUES (met) } \\
\cline { 2 - 6 } & PN & $\begin{array}{l}\text { APN } \\
\text { Perfect target } \\
\text { information }\end{array}$ & $\begin{array}{l}\text { with target maneu- } \\
\text { ver detection and } \\
\text { estimation }\end{array}$ & $\begin{array}{l}\text { (MAMS with detecti- } \\
\text { on and estimation) }\end{array}$ \\
\hline 0.0 & $4.8(3.8)$ & $8.69(4.80)$ & $12.85(7.3)$ & \\
1.0 & $9.9(4.0)$ & $4.40(3.18)$ & $5.43(4.0)$ & \\
2.0 & $6.8(3.7)$ & $3.60(2.90)$ & $4.28(2.7)$ & \\
5.0 & $2.8(2.0)$ & $3.50(2.60)$ & $3.10(2.2)$ & $2.13(2.68)$ \\
7.0 & $16.1(5.0)$ & $3.70(2.75)$ & $7.30(3.7)$ & \\
7.5 & $18.3(5.0)$ & $6.83(2.94)$ & $4.62(3.0)$ & \\
1eve1 & $4.3(2.8)$ & $3.80(2.50)$ & $3.80(2.5)$ & \\
\hline
\end{tabular}

the integrated acceleration square is much smaller in APN. This APN acceleration profile very closely resembles the one obtained from the LQ tracking formulation of guidance problem. While the heading error modifies the initial acceleration profile, it has no pronounced effect on the miss distance.

The results establish that the acceleration profiles are qualitatively different under the two dimensional engagement scenario compared to the one dimensional case presented by Nesline and Zarchan. While APN and LQ guidance schemes involve additional effort in estimating the target acceleration reasonably accurately, the smaller integrated acceleration can be exploited for range advantage.

\section{References}

1. Pastrick, H.L., Seltzer, S.N. and Warren, M.E., 'Guidance Laws for short-range tactical missiles', AIAA J. Guidance and Control, Vol 4, No.2,1981,pp 98-108.

2. Nesline, F.W. and Zarchan P., 'A new look at Classical Vs Modern Homing Missile Guidance', AIAA J. Guidance and Control, Vol 4 No.1, 1981, pP 78-85.

3. Nesline F.W. and Zarchan, P., 'Miss Distance Dynamics in Homing Missiles', AIAA
Guidance and Control conference proceedings, Aug 1984, pp 78-98.

4. Rogers, R.M., 'Sensitivity of higher order guidance law to parameter variations', IEEE NAECON Proceedings, 1982.

5. Fitzgerald T.J., 'Shaping Filters for Distrubances with Random starting times', AIAA J. Guidance and Control, Vol. 2, No. 2, 1979, pp 152-154.

6. Sarma, I.G. and Swamy, K.N., 'MAMS: An approach to Optimal Terminal Homing Guidance for Aerial Engagement', AIAA Guidance, Navigation and Control Conference, Aug 1989 (accepted)

7. Speyer, J.L. and David, G.H., 'Comparison of Several Extended Kalman Filter Formulations for Homing Missile', AIAA Guidance and Control Conference Proceedings, 1980 pp 392-398.

8. Gonzalez, J.M., 'New Methods in the Terminal Guidance and Control of Tactical Missiles', IEEE NAECON Proceedings 1979, pP 350-361.

9. Chan,Y.T.,Hu,A.G.C. and Plant,J.B.,'A Kalman Filter based Tracking Scheme with input estimation.', IEEE Transactions on AES vol. AES-15, March 1979, pp 237-44 